\title{
Mutations in SDCCAG8/NPHP10 Cause Bardet-Biedl Syndrome and Are Associated with Penetrant Renal Disease and Absent Polydactyly
}

\author{
E. Schaefer ${ }^{\mathrm{a}}$ A. Zaloszyc ${ }^{\mathrm{a}} \quad$ J. Lauer ${ }^{\mathrm{a}} \quad$ M. Durand ${ }^{\mathrm{a}}$ F. Stutzmann ${ }^{\mathrm{a}} \quad$ Y. Perdomo-Trujillo ${ }^{\mathrm{b}}$

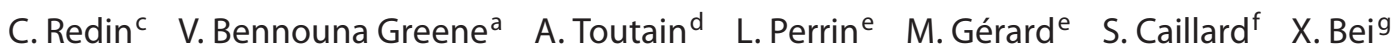

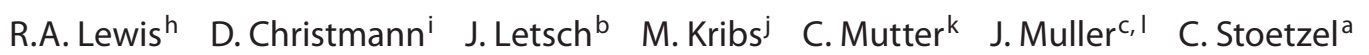 \\ M. Fischbach ${ }^{m}$ V. Marion ${ }^{a} \quad$ N. Katsanis ${ }^{9}$ H. Dollfus ${ }^{a, b}$
}

aLaboratoire de Génétique Médicale EA 3949, Equipe Avenir-Inserm, Faculté de Médecine de Strasbourg, Université de Strasbourg, ${ }^{b}$ Centre de Référence pour les Affections Rares en Génétique Ophtalmologique (CARGO), Hôpitaux Universitaires de Strasbourg, Strasbourg, ' Institut de Génétique et de Biologie Moléculaire et Cellulaire, Département de Neurobiologie et Génétique, Laboratoire de Bioinformatique et Génomique Intégratives, Illkirch-Graffenstaden, d Service de Génétique, Hôpitaux Universitaires de Tours, Tours, e'Département de

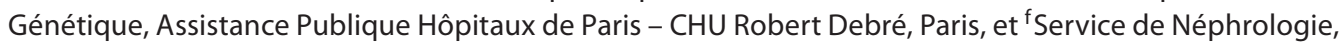
Dialyse et Transplantation Rénale, Hôpitaux Universitaires de Strasbourg, Strasbourg, France; ${ }^{9}$ Center for Human Disease Modeling, Duke University Medical Center, Durham, N.C., and hepartment of Ophthalmology, Baylor College of Medicine, Houston, Tex., USA; 'Service de Radiologie 2, Hôpitaux Universitaires de Strasbourg, Strasbourg, jService de Néphrologie et d'Hémodialyse, Centre Hospitalier Regional, Haguenau,

kCentre d'Investigation Clinique des Hôpitaux Universitaires de Strasbourg, 'Laboratoire de Diagnostic Génétique, Nouvel Hôpital Civil, et ${ }^{\mathrm{m}}$ Service de Pédiatrie 1, Hôpitaux Universitaires de Strasbourg, Strasbourg, France

\section{Key Words}

Bardet-Biedl · Ciliopathy $\cdot$ Nephronophthisis • Polydactyly • SDCCAG8

\begin{abstract}
The ciliopathies are an expanding group of disorders caused by mutations in genes implicated in the biogenesis and function of primary cilia. Bardet-Biedl syndrome (BBS) is a model ciliopathy characterized by progressive retinal degeneration, obesity, polydactyly, cognitive impairment, kidney anomalies and hypogonadism. Mutations in SDCCAG8 (NPHP10) were described recently in patients with nephronophthisis and retinal degeneration (Senior-Loken syndrome; SLS). Given the phenotypic and genetic overlap between known ciliopathy genes, we hypothesized that mutations in SDCCAG8 might also contribute alleles to more
\end{abstract}

\section{KARGER}

(C) 2011 S. Karger AG, Basel

Fax +41613061234 E-Mail karger@karger.ch www.karger.com
Accessible online at: www.karger.com/msy severe, multisystemic ciliopathies. We performed genetic and phenotypic analyses of 2 independent BBS cohorts. Subsequent to mutation screening, we made a detailed phenotypic analysis of 5 families mutated for SDCCAG8 (3 homozygous and 2 compound heterozygous mutations) and conducted statistical analyses across both cohorts to examine possible phenotype-genotype correlations with mutations at this locus. All patients with mutations in SDCCAG8 fulfilled the diagnostic criteria for BBS (retinal degeneration, obesity, cognitive defects, renal failure, hypogonadism). Interestingly, none of the patients with primary SDCCAG8 mutations had polydactyly, a frequent but not obligatory BBS feature. In contrast, the same patients displayed early-onset renal failure, obesity, as well as recurrent pulmonary and ENT in-

\section{E.S. and A.Z. contributed equally to this work.}

Prof. Helene Dollfus

Laboratoire de Génétique Médicale EA 3949 Inserm Avenir

Université de Strasbourg, Hôpitaux Universitaires de Strasbourg

11 rue Humann, FR-67085 Strasbourg Cedex (France)

Tel. +33 3881281 20,E-Mail hdollfus@ unistra.fr 
fections. Comparison of the phenotypes of these families with our entire BBS cohort indicated that renal impairment and absent polydactyly correlated significantly with causal SDCCAG8 mutations. Thus, SDCCAG8 mutations are sufficient to cause BBS in $1-2 \%$ of our combined cohorts, and define this gene as the sixteenth BBS locus (BBS16). The absence of polydactyly and the concomitant, apparently fully penetrant association with early kidney failure represents the first significant genotype-phenotype correlation in BBS that potentially represents an indicator for phenotype-driven priority screening and informs specific patient management.

Copyright $\odot 2011$ S. Karger AG, Basel

The ciliopathies represent a diverse group of clinically distinct but phenotypically overlapping disorders caused by mutations in genes involved in the biogenesis or maintenance of the primary cilium, a sensory organelle found in most mammalian cells [Gerdes et al., 2009]. The phenotypic range of ciliopathies encompasses both single-organ disorders as well as broad syndromic conditions, and their range of onset likewise varies from prenatally to early childhood. To date, mutations in $>30$ genes have been reported to be involved in this spectrum of diseases [Badano et al., 2006]. The ciliopathies are also characterized by a high degree of genetic heterogeneity, with little evidence of genotype-phenotype correlations. For example, mutations in 11 genes have been shown to be genetically necessary to cause nephronophthisis (NPHP, or, when present in conjunction with retinal degeneration, Senior-Loken syndrome, SLS), while 15 causal genes have been identified in Bardet-Biedl syndrome (BBS) [Gascue et al., 2011]. BBS is a model ciliopathy exhibiting characteristic phenotypic pleiotropy, illustrated by 5 major features: retinitis pigmentosa (RP), obesity, polydactyly, urogenital abnormalities, and cognitive impairment [Beales et al., 1999]. BBS genes have been identified through various strategies ranging from traditional positional cloning, in some instances associated with comparative genomics [Katsanis et al., 2000; Slavotinek et al., 2000; Mykytyn et al., 2001, 2002; Nishimura et al., 2001, 2005; Chiang et al., 2004, 2006; Li et al., 2004; Stoetzel et al., 2006, 2007], to homology-based approaches [Ansley et al., 2003; Badano et al., 2003; Fan et al., 2004], evaluation of functional candidates [Kim et al., 2010] and overlap with other clinically distinct ciliopathies [Leitch et al., 2008]. The latest reported retinorenal ciliopathy gene, SDCCAG8, was identified by implementing an exon capture approach and homozygosity mapping with subsequent dedicated sequencing in phenotypically diverse cohorts that included patients with

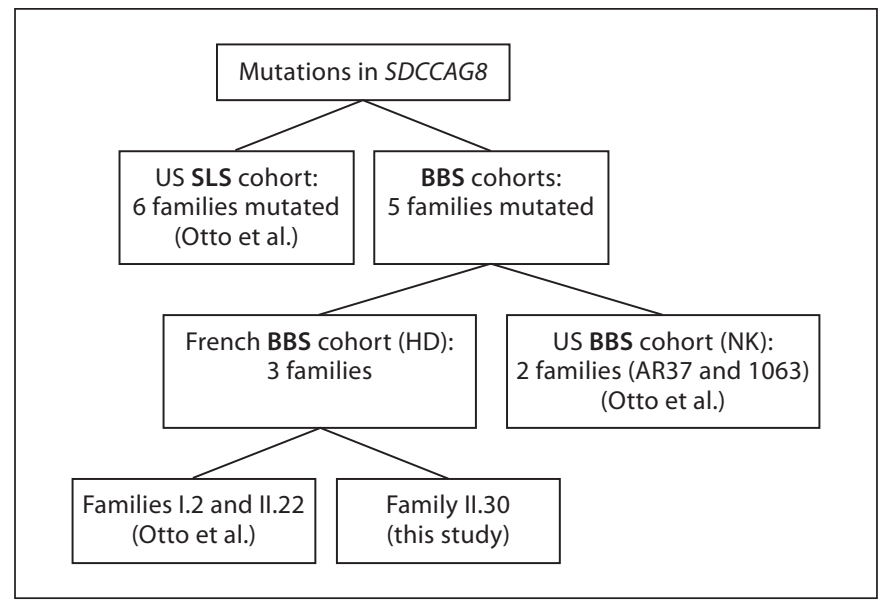

Fig. 1. Representation of families reported with mutations in SDCCAG8 (H.D.: Helene Dollfus; N.K.: Nicholas Katsanis).

NPHP, including the associated SLS phenotype, as well as patients with phenotypes consistent with BBS [Otto et al., 2010]. We describe herein the genetic and phenotypic analysis of BBS patients from 2 large independent BBS cohorts (fig. 1). Overall, we identified 5 families fulfilling the diagnostic criteria for BBS and carrying causal mutations in SDCCAG8. We provide evidence that mutations in this locus are sufficient to cause this disorder, rendering SDCCAG8/NPHP10 the sixteenth BBS gene (BBS16) and expanding the notion that mutations in some ciliary genes can drive the development of diverse clinical phenotypes [Zaghloul and Katsanis, 2009]. Moreover, we show that in each cohort, causal SDCCAG8 mutations lead to absent polydactyly and fully penetrant renal disease, providing the first significant genotype-phenotype correlation and potentially offering predictive value for clinical genetic testing for any of the known BBS loci.

\section{Patients and Methods}

Patients and Related Phenotype

The patients analyzed in this study came from 2 independent BBS cohorts, 1 investigated in France (H.D.) and 1 in the United States (N.K.). Inclusion criteria have been defined elsewhere [Beales et al., 1999]. Informed consent was obtained from all participants in the study, in accord with the tenets of the Declaration of Helsinski.

The French BBS cohort includes more than 400 BBS families collected since 2002. Twenty of them with no apparent mutation in BBS1-BBS14 were selected for a first screen by SNP array analysis because of consanguinity and/or because of the number of affected individuals. After the identification of mutations in 


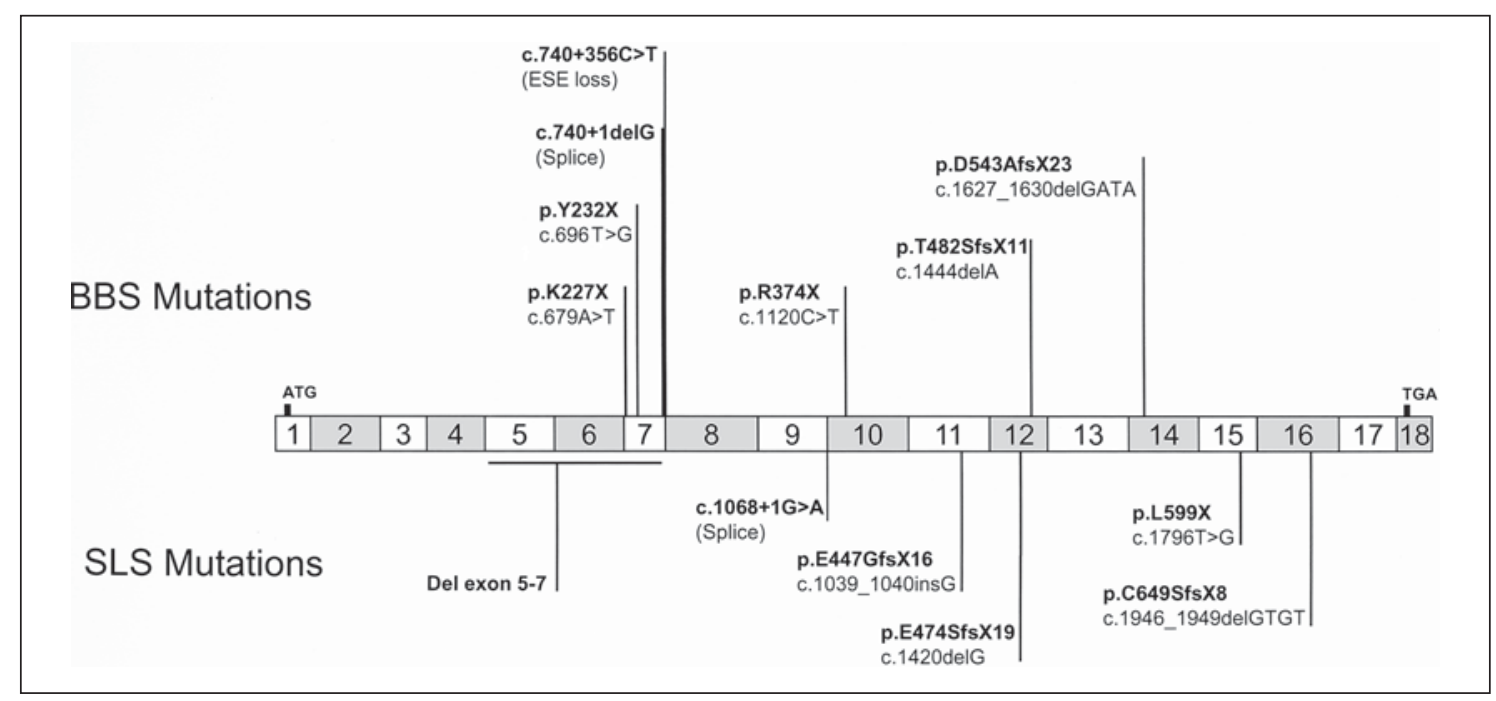

Fig. 2. Mutations reported in SDCCAG8 to date. Mutations described in BBS patients are represented above the gene and mutations identified in SLS patients below. ESE = Exonic splicing enhancer.

SDCCAG8 in 3 families (I.2, II.22 and II.30), a second series of 40 patients, comprising mostly sporadic cases, was tested.

For the US BBS cohort, a series of 94 unrelated patients with BBS was tested irrespective of the presence of mutations at other loci.

\section{Homozygosity Mapping (French Cohort)}

Homozygosity mapping results for families I.2 and II.22 have been described elsewhere [Otto et al., 2010]. Family II.30, reported here for the first time, was genotyped with the Affymetrix GeneChip Human Mapping 250K Nsp Array (Affymetrix, Santa Clara, Calif., USA). Sample processing and labeling were conducted according to the manufacturer's instructions. Genotypes were called with Genotyping Console (GTC v4.0, Affymetrix).

\section{Sequencing}

Genomic DNA was extracted from blood samples according to the manufacturer's protocol (Flexigene DNA kit, Qiagen). PCR amplification was performed with 50 ng of genomic DNA template. The primers were designed with Primer 3 (http://frodo.wi. mit.edu/primer3); detailed protocols and primers are available on request. Bidirectional sequencing of purified PCR products was performed by the GATC Sequencing Facilities (http://www.gatcbiotech.com/en/about-us/gatc.html) or in-house on an ABI3730 Sequencer (Applied Biosystems, Santa Clara, Calif., USA).

\section{Results}

\section{Homozygosity Mapping (French Cohort)}

Results for families I.2 and II.22 have been reported previously [Otto et al., 2010]. In the consanguineous family II.30, we identified large homozygosity segments in- cluding 4 regions that contain known ciliopathy genes (NPHP2/INVS, NPHP6/CEP290, MKS1, and BBS4). However, we found no mutations by direct sequencing of each locus. SDCCAG8 was located in one of the smaller homozygosity regions $(1.4 \mathrm{Mb})$ and, given its candidacy, we sequenced its coding regions. We identified a homozygous nonsense mutation in exon 10 (p.R374X, c.1120C>T) (fig. 2) that was absent from 192 ethnically-matched controls and segregated with the disorder under an autosomal recessive mode of inheritance.

\section{Phenotype and Genotype}

Family II.30 represents the fifth BBS family with mutations in SDCCAG8, in addition to the 6 reported SLS families [Otto et al., 2010] (fig. 1). The genotypic and phenotypic data for each family are summarized in table 1.

The first family, I.2, is a Gypsy consanguineous kinship with 5 affected members found in 2 related sibships. Affected sibs harbored a homozygous deep intronic mutation $(\mathrm{c} .740+356 \mathrm{C}>\mathrm{T})$ in intron 7 that was predicted to lead to the loss of an exonic splicing enhancer site, a prediction confirmed by cDNA sequencing [Zhang et al., 2005; Otto et al., 2010] (fig. 2). Although this family is classified as classic BBS, they were recruited initially because of the acute manifestation of chronic renal failure that was coupled to a variety of respiratory defects: patient I.2.21 was dialyzed between the age of 22 and 25 years, at which point the patient underwent a renal transplantation. Moreover, this patient developed subsequent- 
Table 1. Main clinical manifestations and mutations in SDCCAG8 for the patients reported in this study

\begin{tabular}{|c|c|c|c|c|c|c|c|c|c|c|c|c|}
\hline & \multicolumn{12}{|c|}{ Family } \\
\hline & \multicolumn{5}{|l|}{$\mathrm{I} .2$} & \multicolumn{2}{|l|}{ II. 22} & II. 30 & \multicolumn{3}{|l|}{ AR37 } & 1063 \\
\hline Parental consanguinity & \multicolumn{5}{|l|}{ yes } & \multicolumn{2}{|l|}{ yes } & yes & \multicolumn{3}{|l|}{ no } & no \\
\hline Ethnic origin & \multicolumn{5}{|l|}{ Gypsy } & \multicolumn{2}{|l|}{ Europe } & Turkish & \multicolumn{3}{|c|}{ North European } & India \\
\hline Individual & I. 2.21 & I.2.22 & I.2.23 & I.2.24 & I.2.25 & II.22.21 & II.22.22 & & AR37-02 & AR37-05 & AR37-07 & \\
\hline Gender & M & $\mathrm{F}$ & $\mathrm{F}$ & M & M & M & $\mathrm{F}$ & $\mathrm{F}$ & $\mathrm{M}$ & M & $\mathrm{F}$ & ND \\
\hline Age, years & 37 & 22 & 14 & 8 & 31 & 19 & 15 & 6 & 27 & 22 & 11 & ND \\
\hline Obesity (BMI) & - & $+(38)$ & $+(34)$ & - & $+(54)$ & $+(41)$ & $+(31)$ & $+(22)$ & $+(31)$ & $+(30)$ & $+(28)$ & + \\
\hline Cone-rod dystrophy (age) & $+(29)$ & $+(13)$ & $+(10)$ & $+(6)$ & $+(31)$ & $+(13)$ & $+(9)$ & $+(5)$ & + & $+(13)$ & $+(11)$ & + \\
\hline Chronic renal failure (age) & $+(22)$ & $+(13)$ & $+(10)$ & $+(6)$ & $+(31)$ & $+(13)$ & $+(13)$ & $+(5)$ & $+(28)$ & $+(22)$ & $+(?)$ & ND \\
\hline Polydactyly & - & - & - & - & - & - & - & - & - & - & - & - \\
\hline Hypogonadism/hypogenitalism & ND & ND & ND & ND & + & + & + & ND & ND & + & ND & ND \\
\hline Developmental delay & ND & + & + & + & + & + & + & + & - & - & + & + \\
\hline $\begin{array}{l}\text { Conductive hearing loss/ } \\
\text { recurrent otitis }\end{array}$ & + & + & + & + & ND & + & + & + & ND & ND & ND & ND \\
\hline Respiratory infection & + & + & + & + & + & + & + & + & ND & ND & ND & ND \\
\hline Respiratory failure & + & + & - & - & ND & ND & ND & ND & ND & ND & ND & ND \\
\hline Asthma & + & + & + & + & ND & + & + & $\mathrm{ND}$ & ND & ND & ND & ND \\
\hline Mutation & c. $740+3$ & $356 \mathrm{C}>\mathrm{T} /$ & c. $740+3$ & $56 \mathrm{C}>\mathrm{T}$ & & c. $679 \mathrm{~A}>$ & $/ c .679 A>T$ & $\begin{array}{l}\text { c. } 1120 \mathrm{C}>\mathrm{T} / \\
\mathrm{c} .1120 \mathrm{C}>\mathrm{T}\end{array}$ & c. $696 \mathrm{~T}>\mathrm{G}$ & $/ c .740+1 \mathrm{del}$ & & $\begin{array}{l}\text { c.1444delA/ } \\
\text { c.1627_1630 } \\
\text { delGATA }\end{array}$ \\
\hline Location of mutation & intron & & & & & exon 7 & & exon 10 & exon 7 & & & exon $12 / 14$ \\
\hline Protein modification & loss of & ESE site & (aberran & t ins IV & & p.K227X & p.K227X & $\begin{array}{l}\text { p.R374X/ } \\
\text { p.R374X }\end{array}$ & p.Y232X/ & splice & & $\begin{array}{l}\text { p.T482SfsX11/ } \\
\text { p.D543AfsX23 }\end{array}$ \\
\hline Familial segregation & yes & & & & & $\begin{array}{l}\text { father } h \\
\text { mother }\end{array}$ & & $\begin{array}{l}\text { father htz/ } \\
\text { mother htz }\end{array}$ & ND & & & ND \\
\hline
\end{tabular}

$\mathrm{BMI}=$ Body mass index $\mathrm{c}=$ coding DNA; del = deletion; $\mathrm{ESE}=$ exonic splicing enhancer $; \mathrm{fs}=$ frameshift htz $=$ heterozygous; $\mathrm{IVS}=$ intervening sequence; $\mathrm{ND}=$ not determined; $\mathrm{p}=$ protein .

ly respiratory infections, bronchiectasis, and obstructive chronic respiratory failure, a combination of features seen uncommonly in BBS.

BBS was diagnosed 2 years later as RP was established both for him and his 13-year-old sister (I.2.22) (fig. 3A, fundus). In addition to RP, she exhibited obesity (BMI: 38 $\mathrm{kg} / \mathrm{m}^{2}$ ) (fig. 3A, whole body), mental retardation, and moderate deafness due to recurrent otitis. Renal failure was diagnosed and required dialysis and later transplantation. Like her brother, she presented early with regular respiratory infections and she developed bronchiectasis of the medium lobus and chronic obstructive respiratory failure. Although anosmia has been demonstrated in some $50 \%$ of BBS patients [Kulaga et al., 2004], she was normosmic, while a nasal biopsy showed ciliary normal beating.

The third affected sib (patient I.2.23) was regularly admitted to hospital since birth because of respiratory infections and delayed cognitive skills. The recurrent respi- ratory and ENT infections led to a chronic obstructive broncho-pneumopathy and to moderate conductive hearing loss. A CT scan revealed bronchiectasis. Immune deficiency, cystic fibrosis and allergy were excluded and a nasal biopsy showed normal motile cilia. An abdominal ultrasound showed hyperechogenic kidneys at 1 year of life; at age 9, kidneys were consistently hyperechogenic, with enlarged cortex and abnormal cortico-medullary differentiation. Renal scintigraphy showed mildly impaired renal function. RP was subsequently diagnosed, fulfilling the diagnostic criteria for BBS. One year later, the patient suffered from terminal renal failure and the ultrasound showed bilateral renal hypotrophy $(55 \times 23$ $\mathrm{mm}$ ) that required dialysis and renal transplantation 3 years later. Consistent with BBS, the patient became progressively obese (BMI: $34 \mathrm{~kg} / \mathrm{m}^{2}$ ). She was evaluated on the cognitive level by the Wechsler Intelligence Scale for Children ${ }^{\circledR}$ (WISC ${ }^{\circledR}$-IV) and showed moderate cognitive impairment and mental retardation (data not shown). 

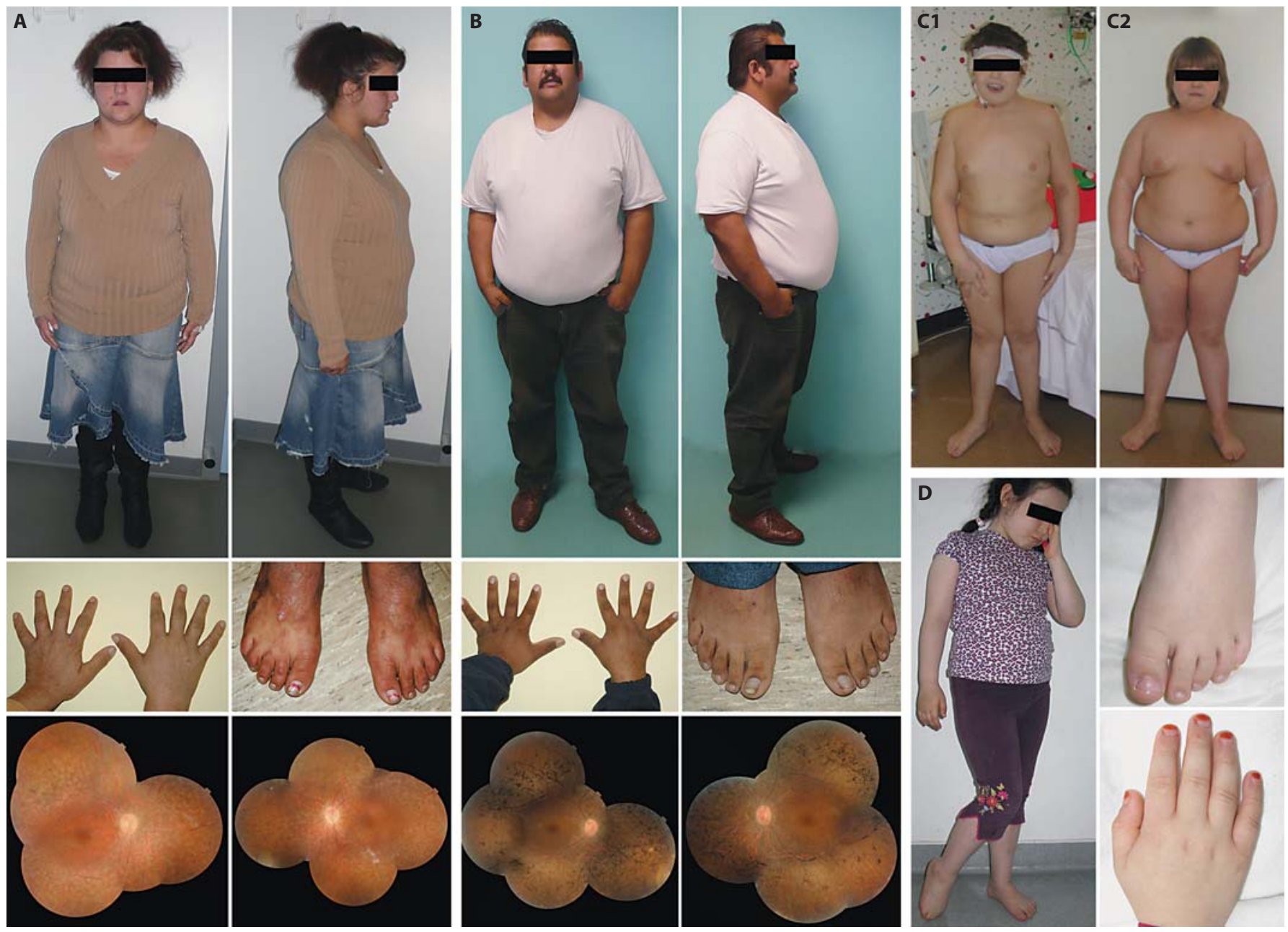

Fig. 3. A, B Photographs of patients I.2.22 (A) and I.2.25 (B) showing the obesity (photograph from head to toe), normal extremities and retinitis pigmentosa (lower fundus photographs), respectively. C Photographs of patients II.22.21 (C1) and II.22.22 (C2) showing obesity with genu valgum orthopedic complications. D Photographs of patient II.30 showing excess of weight and normal extremities.

Her brother (patient I.2.24) was diagnosed with terminal renal failure requiring dialysis at 6 years of age. An ultrasound revealed small kidneys $(60 \times 24 \mathrm{~mm})$ with a complete undifferentiated parenchyma. Ophthalmic examination identified RP. Mental retardation was also diagnosed. Similar to the other affected members of this family, the patient was also treated regularly for bronchial and pulmonary infections and recurrent otitis with moderate conductive hearing loss.

Patient I.2.25 was the eldest at the time of BBS diagnosis. In fact, with the exception of several pulmonary infections and morbid obesity (BMI: $54 \mathrm{~kg} / \mathrm{m}^{2}$ ) (fig. 3B, whole body), the patient had no other complaint. At the age of 31 years, he presented with acute respiratory dis- tress and edema of the lower limbs. Terminal renal failure was diagnosed and required dialysis. The ultrasound showed atrophic kidneys (100 mm of maximum height) with a thin cortex and a bad cortico-medullary differentiation. The diagnosis of RP was made at the same age, although the patient, with mild cognitive impairment, had not noticed that his visual fields were tubular (fig. 3B, fundus).

The combination of our clinical examination secured a diagnosis of BBS for this family. All affected sibs met the criteria for BBS although they all lacked polydactyly, one of the features of BBS that, in some populations, is present in $>60 \%$ of patients [Beales et al., 1999] (fig. 3A, B, hands and feet; fig. 4). 

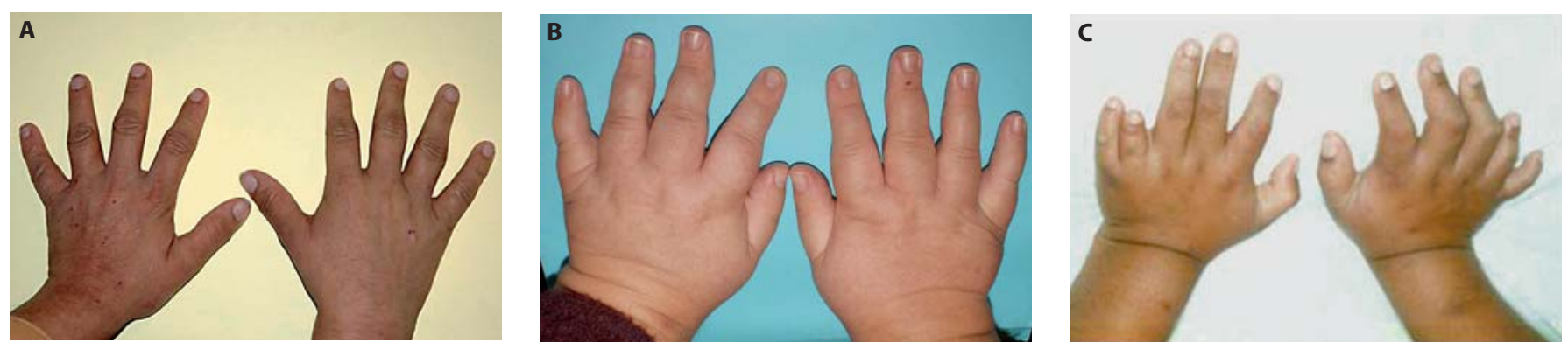

Fig. 4. Photographs showing different types of extremities found in Bardet-Biedl syndrome. A Normal extremities for an SDCCAG8 mutated patient. B Typical brachydactyly found in BBS (here: BBS10-mutated patient) [Stoetzel et al., 2006]. C Typical polydactyly found in BBS (here: BBS12-mutated patient) [Stoetzel et al., 2007].

The second family II.22 is a French consanguineous family. The affected sibs carried a homozygous nonsense mutation (p.K227X, c.679A >T) in exon 7 of SDCCAG8 [Otto et al., 2010] (fig. 2). Terminal renal failure was diagnosed for patient II.22.21 at 13 years of age. The kidney ultrasound was normal, except for several small cysts. Dialysis began immediately and he received a renal transplantation. The clinical association of renal disease with obesity (BMI: $28 \mathrm{~kg} / \mathrm{m}^{2}$; $+5 \mathrm{SD}$ ) (fig. $3 \mathrm{Cl}$ ) and cognitive impairment led to a suspected diagnosis of BBS, despite the lack of polydactyly. This patient also had asthma and recurrent ENT infections with adenoidectomy and myringotomy at age 2. Follow-up showed a significant increase of obesity, with a BMI of $40 \mathrm{~kg} / \mathrm{m}^{2}$ at adult age. His 9-year-old sister II.22.22 had no polydactyly but obesity with a BMI of $31 \mathrm{~kg} / \mathrm{m}^{2}$ (+10 SD) (fig. 3C2), cognitive impairment and respiratory/ENT manifestations. For both of them, an extensive medical investigation also revealed $\mathrm{RP}$, hypogenitalism (unilateral cryptorchism, small penis and testicles for the boy and labia minora hypoplasia for the girl), substantiating the BBS diagnosis. The ultrasound of patient II.22.22 showed kidneys of normal size but with bilateral cortico-medullary dedifferentiation. Follow-up detected progressive renal deterioration requiring dialysis, followed by subsequent transplantation.

The family II. 30 proband is a sporadic case of consanguineous Turkish lineage, carrying a homozygous nonsense mutation (p.R374X, c.1120C >T) in SDCCAG8 (identified in this study; fig. 2). Since the age of 1 month, she presented recurrent respiratory infections and bronchiolitis, leading to the diagnosis of infantile asth ma. She also presented recurrent ENT infections and benefited from adenoidectomy and myringotomy. Moreover, she developed early-onset obesity (from month 6) and weighed $27.6 \mathrm{~kg}$ for a height of $111 \mathrm{~cm}$ at the age of 4 (BMI:
$22.4 \mathrm{~kg} / \mathrm{m}^{2} ;+6 \mathrm{SD}$ ) (fig. 3D, whole body). At 5 years of age, she was hospitalized for general deterioration related to terminal renal failure. She was dialyzed and received a renal transplant a few months later. The histological renal evaluation suggested nephronophthisis. RP was diagnosed on the ERG, although the ocular fundus was unremarkable. Although an initial diagnosis of SLS was consistent with her findings (with obesity possibly being coincidental), she also had mild mental retardation and speech delay, which, in spite of the absence of polydactyly (fig. 3D, hand and feet), suggested BBS. She is, to date, the youngest patient affected in our cohort.

The sequencing of 96 unrelated patients of the US cohort led to the identification of 2 families with mutations in SDCCAG8. Family AR37 is a non-consanguineous family of North-European descent with 3 affected sibs and 3 unaffected sibs. Affected sibs harbored 2 compound mutations in exon 7: a nonsense mutation (p.Y232X, c.696T >G) and a splice site mutation (c.740+ 1delG) [Otto et al., 2010] (fig. 2).

The 3 sibs were obese, with a BMI of $28 \mathrm{~kg} / \mathrm{m}^{2}(+5 \mathrm{SD})$ for the youngest (AR37-07; 11 years old), $30 \mathrm{~kg} / \mathrm{m}^{2}$ for the second (AR37-05; 22 years old) and $31 \mathrm{~kg} / \mathrm{m}^{2}$ for the eldest (AR37-02; 27 years old). All of them had RP, diagnosed at 13 and 11 years old for AR37-05 and AR37-07, respectively (data on first diagnosis are not available for AR37-02). Similar to the patients in the French cohort, patients AR37-02 and -05 had terminal renal failure diagnosed at 28 and 22 years, respectively. The renal ultrasound of patient AR37-07 at 12 years showed early renal disease; the kidney biopsy revealed tubular and glomerular disease with persistent fetal glomeruli, prompting rigorous kidney follow-up. Notably, again reminiscent of the patients with SDCCAG8 mutations in the French cohort, none of the 3 affected sibs had polydactyly. One patient 
had hypogenitalism (AR37-05) and one had cognitive impairment (AR37-07).

Finally, 2 heterozygous deletions causing frameshifts and inducing a premature stop codon were identified in exon 12 (p.T482SfsX11, c.1444delA) and in exon 14 (p.D543AfsX23, c.1627_1630delGATA) in a sporadic case born from Indian non-consanguineous parents (F1063) [Otto et al., 2010] (fig. 2). This boy presented terminal renal failure in childhood, RP, and developmental delay, but no polydactyly. No more clinical data are available as the family is no longer accessible for follow-up studies.

\section{First Evidence for Gene-Specific Phenotypes in} BBS Patients

Despite the documented clinical variability of BBS, we were intrigued by the consistent presence of renal disease with a concomitant absence of polydactyly in all BBS patients in which SDCCAG8 mutations were sufficient to explain the disorder. We therefore wondered whether this pattern was significant in our cohorts. Because the number of SDCCAG8-positive patients is modest, we compared: (a) the frequency of the single occurrence of either renal disease or polydactyly in SDCCAG8 patients versus our cohorts; (b) the recurrence of these phenotypes in sib pairs with or without SDCCAG8 mutations, and (c) the frequency of the combined occurrence of renal failure along with absence of polydactyly in SDCCAG8 patients versus the cohorts.

In the French cohort, the 8 SDCCAG8-positive BBS patients all shared the same phenotype: renal failure $(8 / 8)$, but no polydactyly $(8 / 8)$, whereas in the 36 SDCCAG8-negative BBS patients only 18/36 had renal failure, and 15 of them (15/18) showed polydactyly. In the US cohort, we found a low incidence of renal disease: 12/92 SDCCAG8-negative patients had renal disease versus 4/4 SDCCAG8-positive, a significant difference (Fisher's exact test: $\mathrm{p}<0.0005)$. These data reveal a significant difference of phenotype between SDCCAG8-positive patients and the rest of the BBS cohorts, at both levels of renal failure feature $(\mathrm{p}<0.0089)$ or absence of polydactyly $(\mathrm{p}<0.0042)$.

Consistent with the above data, in the US cohort the intrafamilial recurrence of renal disease was absolute in our 3 SDCCAG8-positive sib pairs, but true only in 2/37 negative sib pairs, an observation that was also significant $(\mathrm{p}<0.0011)$ and which strengthened the hypothesis of renal disease recurrence being higher in SDCCAG8positive sib pairs than in negative ones.

Finally, when we asked for the co-occurrence of renal disease and absence of polydactyly, we found an even more striking (and significant) difference. Once again, in the French PHRC 2002 cohort we identified 3 out of the 36 SDCCAG8-negative patients without polydactyly and with renal impairment, whereas $100 \%$ of the SDCCAG8 patients with renal failure had no polydactyly $(\mathrm{p}<0.0001)$. Identically, whereas all the US patients (and sib pairs) were fully concordant with these phenotypes, we only found 4/92 SDCCAG8-negative patients with renal disease and no polydactyly $(\mathrm{p}<$ $0.00002)$ and $0 / 37$ sib pairs $(p<0.0001)$. The data for both cohorts only show a significant absence of polydactyly in renal affected SDCCAG8 patients as compared to renal affected SDCCAG8-negative patients. In the French cohort, the co-occurrence of renal failure and absence of polydactyly in all SDCCAG8 patients versus the rest of the BBS cohorts was also found to be significant $(p<0.000004)$

\section{Discussion}

The ciliopathies represent useful models to study the architecture of human genetic disease, especially with regard to the mechanistic underpinnings of genetic heterogeneity and the molecular basis of phenotypic variability. Some exceptions notwithstanding, allelism at single ciliopathy loci is insufficient to explain phenotypic differences, an observation attributed in part to the concept of the variable activity of trans-acting modifiers within the biological system: the primary cilium [Khanna et al., 2009; Zaghloul and Katsanis, 2010; Davis et al., 2011]. For example, loss-of-function mutations in the pan-ciliopathy locus CEP290 can cause isolated nephronophthisis (NPHP6 [Sayer et al., 2006]), SLS (SLSN6 [Sayer et al., 2006]), Leber congenital amaurosis (LCA10 [den Hollander et al., 2006]), BBS (BBS14 [Leitch et al., 2008]), Joubert syndrome (JBTS5 [Sayer et al., 2006]) and MeckelGruber syndrome (MKS4 [Baala et al., 2007]), with no evidence for single locus genotype-phenotype correlations.

The data emerging from the novel ciliopathy gene SDCCAG8 suggest similar phenotypic overlap. While Otto et al. [2010], focusing on a cohort of patients with retinal-kidney phenotypes, identified mutations in SDCCAG8 primarily in patients with an SLS diagnosis, we identified independently mutations in the same transcript in BBS patients. Given the pattern of variation detected in CEP290, as well as in other ciliopathy loci, we predict that SDCCAG8 is an attractive functional/genetic candidate for other related ciliopathies, most notably 
Meckel-Gruber syndrome and Joubert syndrome. However, in contrast to significantly divergent phenotypes seen upon loss of function of other ciliopathy transcripts, all null alleles in SDCCAG8 are concomitant with early renal failure and RP. In most cases in our present study, renal failure was the first symptom of the disease, was discovered at the terminal stage and typically predated the diagnosis of BBS. Notably, although renal impairment represents one of the primary features of BBS, its penetrance ranges from 5 to $82 \%$ [Beales et al., 1999; Imhoff et al., 2011]. In the cohort described by Beales et al. [1999], 6 patients (5\%) presented chronic renal failure and 4 of them were children. Moreover, although all individuals from a series of 20 BBS patients in Newfoundland (mean age: 30 years) presented structural and/or functional renal abnormalities, only 3 (15\%) experienced terminal renal failure [Harnett et al., 1988]. As such, it is unusual that both the large US and French BBS cohorts used in the present study show a statistically significant and apparently fully penetrant end stage kidney disease in SDCCAG8 patients (100\%) as opposed to a low incidence of kidney failure in the SDCCAG8-negative BBS patients (13 and $8 \%$, respectively).

In addition to the renal phenotype, 10 of 12 SDCCAG8 BBS patients $(83 \%)$ presented early-onset obesity. Interestingly, a recent meta-analysis of genome-wide association studies for early-onset obesity identified 3 polymorphisms in introns 6, 9 and 10 of SDCCAG8 in strong pairwise linkage disequilibrium [Scherag et al., 2010]. This association of SDCCAG8 variants with pediatric obesity prior to the discovery of its involvement in BBS mirrors that of $B B S 2, B B S 4$ and $B B S 6$ with the association of their polymorphisms with childhood and late-onset obesity reported after their BBS-causing mutations [Benzinou et al., 2006]. Taken together, these findings suggest that (a) BBS- and other ciliopathy-causing genes might be strong candidates for sporadic, genetically complex obesity, and (b) that the underlying obesity mechanism of BBS might be relevant to a broader subset of complex obesity.

All patients in the French cohort also presented recurrent pulmonary and ENT infections in early childhood, symptoms also found in Alström syndrome (ALMS). However, the SDCCAG8 phenotype can be readily distinguished from ALMS as dilated or restrictive cardiomyopathy, diabetes and sensorineural component were not present in any of our patients, while, at the same time, ALMS patients are not known to have cognitive impairment [Alström et al., 1959].

The most striking differential clinical feature in SDCCAG8-BBS patients was the absence of polydactyly, one of the secondary features of BBS. Polydactyly is present in $58-74 \%$ of BBS patients [Bell, 1958; Green et al., 1989] and brachydactyly is often associated [Rudling et al., 1996]. In the French BBS cohort, polydactyly is significantly more common, with $98 / 110$ patients with mutations in BBS1-BBS12 having at least 1 extra digit, and at least 4 more families manifesting brachydactyly. This might represent a peculiarity of the genetic pool from which our patients are enrolled, or, more likely, might represent overly stringent criteria for securing a BBS diagnosis. Irrespective of the source of this difference, the high occurrence of polydactyly in the French cohort renders the absence of this phenotype from all SDCCAG8BBS patients even more striking.

BBS, like most ciliopathies, is largely bereft of genotype-phenotype correlations. The only previously proposed correlation is the association of $B B S 3$ mutations with polydactyly of the 4 limbs [Sheffield et al., 1994] or of the lower limbs [Young et al., 1998]. However, this observation had not been supported by the description of additional patients mutated in BBS3 presenting no polydactyly [Ghadami et al., 2000]. Therefore, our data potentially represent the first significant genotype-phenotype correlation in BBS. We suggest that in absence of polydactyly and brachydactyly in BBS patients SDCCAG8 screening could be important after initial screening for recurrent BBS mutations [Muller et al., 2010], especially when such patients also present acute renal disease and recurrent respiratory/ENT infections. Moreover, the biological underpinning of these differences has the potential to apprehend the mechanism of the development and maintenance of several physiological systems.

\section{Acknowledgements}

We warmly thank the patients who accepted the publication of their clinical data. We thank in particular the French BardetBiedl support group (association Bardet-Biedl France). We also thank all medical contributors from genetic, pediatric, radiologic, biologic units as well as the CIC of Strasbourg University Hospital. We are grateful to the Centre National de Genotypage of Evry and the Affymetrix platform of IGBMC/Genopole de Strasbourg, especially Christelle Thibault and Violaine Alunni. The work of the French authors is funded by Retina France, UNADEV (Union Nationale des Aveugles et Déficients Visuels), FORMICOEUR, PHRC (Programme Hospitalier pour la Recherche Clinique) program 2007 and the Agence Nationale pour la Recherche call for rare diseases 2009. This study was supported in parts by the National Institutes of Health grants R01HD04260 from the National Institute of Child Health and Development and R01DK072301 from the National Institute of Diabetes, Digestive and Kidney disorders (N.K.). N.K. is a Distinguished Brumley Professor. 


\section{References}

Alström CH, Hallgren B, Nilsson LB, Asander $\mathrm{H}$ : Retinal degeneration combined with obesity, diabetes mellitus and neurogenous deafness: a specific syndrome (not hitherto described) distinct from the LaurenceMoon-Bardet-Biedl syndrome: a clinical, endocrinological and genetic examination based on a large pedigree. Acta Psychiatr Neurol Scand Suppl 129:1-35 (1959).

-Ansley SJ, Badano JL, Blacque OE, Hill J, Hoskins $\mathrm{BE}$, et al: Basal body dysfunction is a likely cause of pleiotropic Bardet-Biedl syndrome. Nature 425:628-633 (2003).

- Baala L, Audollent S, Martinovic J, Ozilou C, Babron MC, et al: Pleiotropic effects of CEP290 (NPHP6) mutations extend to Meckel syndrome. Am J Hum Genet 81:170179 (2007).

Badano JL, Ansley SJ, Leitch CC, Lewis RA, Lupski JR, Katsanis N: Identification of a novel Bardet-Biedl syndrome protein, BBS7, that shares structural features with BBS1 and BBS2. Am J Hum Genet 72:650-658 (2003).

-Badano JL, Mitsuma N, Beales PL, Katsanis N: The ciliopathies: an emerging class of human genetic disorders. Annu Rev Genomics Hum Genet 7:125-148 (2006).

Beales PL, Elcioglu N, Woolf AS, Parker D, Flinter FA: New criteria for improved diagnosis of Bardet-Biedl syndrome: results of a population survey. J Med Genet 36:437-446 (1999).

Bell J: The Laurence-Moon syndrome; in Penrose LS (ed): The Treasury of Human Inheritance, pp 51-96 (Cambridge University Press, Cambridge 1958).

- Benzinou M, Walley A, Lobbens S, Charles MA, Jouret B, et al: Bardet-Biedl syndrome gene variants are associated with both childhood and adult common obesity in French Caucasians. Diabetes 55:2876-2882 (2006).

-Chiang AP, Nishimura D, Searby C, Elbedour K, Carmi R, et al: Comparative genomic analysis identifies an ADP-ribosylation factor-like gene as the cause of Bardet-Biedl syndrome (BBS3). Am J Hum Genet 75:475-484 (2004).

-Chiang AP, Beck JS, Yen HJ, Tayeh MK, Scheetz TE, et al: Homozygosity mapping with SNP arrays identifies TRIM32, an E3 ubiquitin ligase, as a Bardet-Biedl syndrome gene (BBS11). Proc Natl Acad Sci USA 103:62876292 (2006).

- Davis EE, Zhang Q, Liu Q, Diplas BH, Davey LM, et al: TTC21B contributes both causal and modifying alleles across the ciliopathy spectrum. Nat Genet 43:189-196 (2011).

den Hollander AI, Koenekoop RK, Yzer S, Lopez I, Arends ML, et al: Mutations in the CEP290 (NPHP6) gene are a frequent cause of Leber congenital amaurosis. Am J Hum Genet 79: 556-561 (2006).

-Fan Y, Esmail MA, Ansley SJ, Blacque OE, Boroevich $\mathrm{K}$, et al: Mutations in a member of the Ras superfamily of small GTP-binding proteins causes Bardet-Biedl syndrome. Nat Genet 36:989-993 (2004).

- Gascue C, Katsanis N, Badano JL: Cystic diseases of the kidney: ciliary dysfunction and cys- togenic mechanisms. Pediatr Nephrol 26: 1181-1195 (2011).

Gerdes JM, Davis EE, Katsanis N: The vertebrate primary cilium in development, homeostasis, and disease. Cell 137:32-45 (2009).

Ghadami M, Tomita HA, Najafi MT, Damavandi E, Farahvash MS, et al: Bardet-Biedl syndrome type 3 in an Iranian family: clinical study and confirmation of disease localization. Am J Med Genet 94:433-437 (2000).

Green JS, Parfrey PS, Harnett JD, Farid NR, Cramer BC, et al: The cardinal manifestations of Bardet-Biedl syndrome, a form of LaurenceMoon-Biedl syndrome. N Engl J Med 321: 1002-1009 (1989).

-Harnett JD, Green JS, Cramer BC, Johnson G, Chafe L, et al: The spectrum of renal disease in Laurence-Moon-Biedl syndrome. N Engl J Med 319:615-618 (1988).

Imhoff O, Marion V, Stoetzel C, Durand M, Holder M, et al: Bardet-Biedl syndrome: a study of the renal and cardiovascular phenotypes in a French cohort. Clin J Am Soc Nephrol 6:22-29 (2011).

Katsanis N, Beales PL, Woods MO, Lewis RA, Green JS, et al: Mutations in MKKS cause obesity, retinal dystrophy and renal malformations associated with Bardet-Biedl syndrome. Nat Genet 26:67-70 (2000)

Khanna H, Davis EE, Murga-Zamalloa CA, Estrada-Cuzcano A, Lopez I, et al: A common allele in RPGRIPIL is a modifier of retinal degeneration in ciliopathies. Nat Genet 41: 739-745 (2009).

- Kim SK, Shindo A, Park TJ, Oh EC, Ghosh S, et al: Planar cell polarity acts through septins to control collective cell movement and ciliogenesis. Science 329:1337-1340 (2010).

Kulaga HM, Leitch CC, Eichers ER, Badano JL, Lesemann A, et al: Loss of BBS proteins causes anosmia in humans and defects in olfactory cilia structure and function in the mouse. Nat Genet 36:994-998 (2004).

Leitch CC, Zaghloul NA, Davis EE, Stoetzel C, Diaz-Font A, et al: Hypomorphic mutations in syndromic encephalocele genes are associated with Bardet-Biedl syndrome. Nat Genet 40:443-448 (2008).

Li JB, Gerdes JM, Haycraft CJ, Fan Y, Teslovich $\mathrm{TM}$, et al: Comparative genomics identifies a flagellar and basal body proteome that includes the BBS5 human disease gene. Cell 117:541-552 (2004).

Muller J, Stoetzel C, Vincent M, Leitch C, Laurier $\mathrm{V}$, et al: Identification of 28 novel mutations in the Bardet-Biedl syndrome genes: the burden of private mutations in an extensively heterogeneous disease. Hum Genet 127:583-593 (2010).

Mykytyn K, Braun T, Carmi R, Haider NB, Searby $\mathrm{CC}$, et al: Identification of the gene that, when mutated, causes the human obesity syndrome BBS4. Nat Genet 28:188-191 (2001).

-Mykytyn K, Nishimura DY, Searby CC, Shastri $M$, Yen HJ, et al: Identification of the gene (BBS1) most commonly involved in Bardet-
Biedl syndrome, a complex human obesity syndrome. Nat Genet 31:435-438 (2002).

Nishimura DY, Searby CC, Carmi R, Elbedour $\mathrm{K}$, Van Maldergem L, et al: Positional cloning of a novel gene on chromosome $16 \mathrm{q}$ causing Bardet-Biedl syndrome (BBS2). Hum Mol Genet 10:865-874 (2001).

Nishimura DY, Swiderski RE, Searby CC, Berg EM, Ferguson AL, et al: Comparative genomics and gene expression analysis identifies $B B S 9$, a new Bardet-Biedl syndrome gene. Am J Hum Genet 77:1021-1033 (2005).

Otto EA, Hurd TW, Airik R, Chaki M, Zhou W, et al: Candidate exome capture identifies mutation of SDCCAG8 as the cause of a retinal-renal ciliopathy. Nat Genet 42:840-850 (2010).

-Rudling O, Riise R, Tornqvist K, Jonsson K: Skeletal abnormalities of hands and feet in Laurence-Moon-Bardet-Biedl (LMBB) syndrome: a radiographic study. Skeletal Radiol 25:655-660 (1996).

- Sayer JA, Otto EA, O’Toole JF, Nurnberg G, Kennedy MA, et al: The centrosomal protein nephrocystin-6 is mutated in Joubert syndrome and activates transcription factor ATF4. Nat Genet 38:674-681 (2006).

-Scherag A, Dina C, Hinney A, Vatin V, Scherag $S$, et al: Two new loci for body-weight regulation identified in a joint analysis of genomewide association studies for early-onset extreme obesity in French and German study groups. PLoS Genet 6:e1000916 (2010).

- Sheffield VC, Carmi R, Kwitek-Black A, Rokhlina T, Nishimura D, et al: Identification of a Bardet-Biedl syndrome locus on chromosome 3 and evaluation of an efficient approach to homozygosity mapping. Hum Mol Genet 3:1331-1335 (1994).

Slavotinek AM, Stone EM, Mykytyn K, Heckenlively JR, Green JS, et al: Mutations in MKKS cause Bardet-Biedl syndrome. Nat Genet 26: 15-16 (2000).

-Stoetzel C, Laurier V, Davis EE, Muller J, Rix S, et al: BBS10 encodes a vertebrate-specific chaperonin-like protein and is a major BBS locus. Nat Genet 38:521-524 (2006).

- Stoetzel C, Muller J, Laurier V, Davis EE, Zaghloul NA, et al: Identification of a novel BBS gene (BBS12) highlights the major role of a vertebrate-specific branch of chaperonin-related proteins in Bardet-Biedl syndrome. Am J Hum Genet 80:1-11 (2007).

- Young TL, Woods MO, Parfrey PS, Green JS, O'Leary E, et al: Canadian Bardet-Biedl syndrome family reduces the critical region of BBS3 (3p) and presents with a variable phenotype. Am J Med Genet 78:461-467 (1998).

Zaghloul NA, Katsanis N: Mechanistic insights into Bardet-Biedl syndrome, a model ciliopathy. J Clin Invest 119:428-437 (2009).

Zaghloul NA, Katsanis N: Functional modules, mutational load and human genetic disease. Trends Genet 26:168-176 (2010)

-Zhang XH, Kangsamaksin T, Chao MS, Banerjee JK, Chasin LA: Exon inclusion is dependent on predictable exonic splicing enhancers. Mol Cell Biol 25:7323-7332 (2005). 